\title{
Wolf-Rayet star parameters from spectral analyses
}

\author{
Wolf-Rainer Hamann ${ }^{1}$, Götz Gräfener ${ }^{1}$, and Lars Koesterke ${ }^{2}$ \\ ${ }^{1}$ Lehrstuhl Astrophysik, Institut für Physik, Universität Potsdam, \\ Am Neuen Palais 10, D-14469 Potsdam, BRD \\ ${ }^{2}$ NASA Goddard Space Flight Center, Greenbelt MD 20771, USA
}

\begin{abstract}
The Potsdam non-LTE code for expanding atmospheres, which accounts for clumping and iron-line blanketing, has been used to establish a grid of model atmospheres for WC stars. A parameter degeneracy is discovered for early-type WC models which do not depend on the 'stellar temperature'. 15 Galactic WC4-7 stars are analyzed, showing a very uniform carbon abundance (He:C $=55: 40$ ) with only few exceptions.
\end{abstract}

\section{A grid of models for WC stars}

The Potsdam non-LTE code, which accounts for complex model atoms, irongroup line blanketing and clumping ( $c f$. Gräfener et al. 2002), has been employed to establish a grid of WC-type model atmospheres. The following parameters are kept constant for the whole grid: chemical composition He:C:O:Fe $=55: 40: 5: 0.16$ (by mass), luminosity $\log L / \mathrm{L}_{\odot}=5.3$, terminal wind velocity $v_{\infty}=2000 \mathrm{~km} \mathrm{~s}^{-1}$, clumping factor $D=10$. Grid variables are the stellar (effective) temperature $T_{*}$ (referring to $\tau_{\text {Ross }}=20$ ), and the 'transformed radius' $R_{\mathrm{t}}=R_{*}\left[v_{\infty} /(\dot{M} \sqrt{D})\right]^{2 / 3}$ (unit convention: $v_{\infty}$ in $2500 \mathrm{~km} \mathrm{~s}^{-1}, \dot{M}$ in $10^{-4} \mathrm{M}_{\odot} \mathrm{yr}^{-1}, c f$. Hamann \& Koesterke 1998). For a fixed $T_{*}$, emission line equivalent widths of WR models depend to a good approximation only on $R_{\mathrm{t}}$ irrespective of different combinations of $R_{*}$, $\dot{M}, v_{\infty}$ and $D$, while absolute fluxes scale with $R_{*}^{2}$.

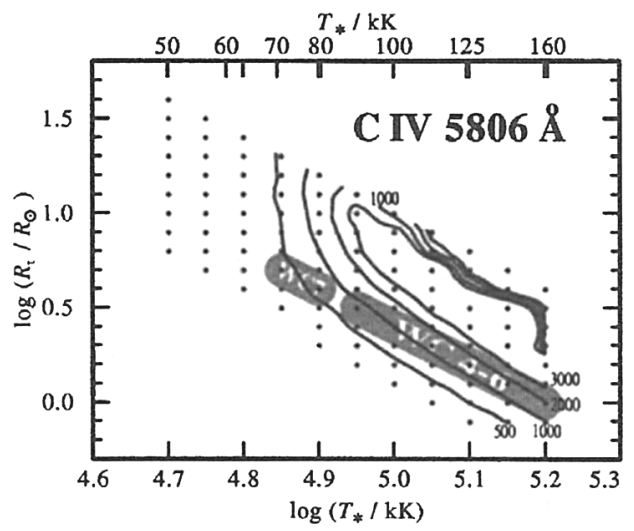

Figure 1. Grid of WC models: contours of equivalent widths for one specific spectral line (labels: $\left.\quad-W_{\lambda} / \AA\right)$. Small circles mark the calculated grid models. The grey bars indicate the location of the analyzed Galactic WC 4-6 $\left(T_{*} \geq 90 \mathrm{kK}\right)$ and WC 7 $\left(T_{*} \lesssim 80 \mathrm{kK}\right)$ stars. Note that for the hotter part the contours almost align with the grey strip. 


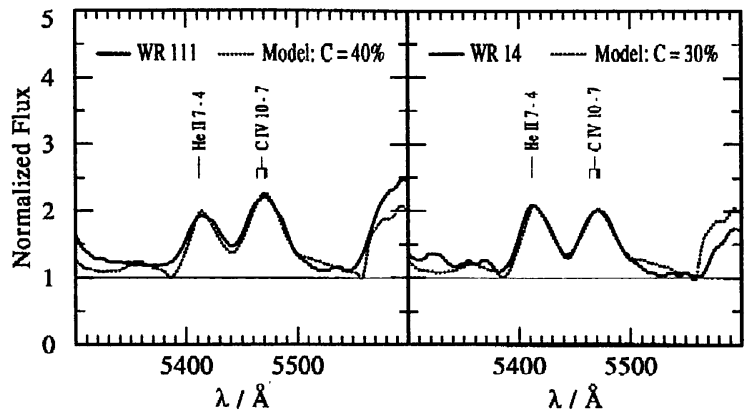

Figure 2. Determination of the carbon abundance from $\mathrm{He}$ II 5412 and Civ 5471. The observed spectrum (full line) of WR 111 is fitted by a model (dotted line) with $\mathrm{He}: \mathrm{C}=55: 40$ (by mass), while WR 14 shows less carbon (right panel).

The contours of equivalent widths in the $\log T_{*}-\log R_{t}$-plane (e.g. Figure 1 ) reveal a further degeneracy for hot, dense expanding atmospheres. For $T_{*} \gtrsim$ $100 \mathrm{kK}$, the contours almost align with $R_{\mathrm{t}} \propto T_{*}^{-2}$ (cf. the grey strip in Figure 1). This means that early-type WC models basically depend on one single parameter only, which we may define as

$$
R_{\mathrm{t} 100 \mathrm{kK}}=R_{\mathrm{t}}\left(\frac{T_{*}}{100 \mathrm{kK}}\right)^{2}, \quad \text { implying } \quad R_{\mathrm{t} 100 \mathrm{kK}} \propto L^{1 / 2}\left(\frac{\dot{M} \sqrt{D}}{v_{\infty}}\right)^{-2 / 3} .
$$

Inspection of synthetic spectra confirms that they are indeed very similar for models with the same $R_{\mathrm{t} 100 \mathrm{kK}}$, irrespective of $T_{*}$. The physical reason is that in dense winds, all radiation (including continuum) arises from rapidly moving layers, and their location is determined by $\dot{M}$ alone. Note that the same $R_{\mathrm{t} 100 \mathrm{kK}}$ implies the same ratio $L / \dot{M}^{4 / 3}\left(v_{\infty}, D\right.$ fixed).

\section{Grid analysis of Galactic WC stars (12 WC 4-6 and 3 WC 7)}

Spectra of 15 (putatively) single WC 4-7 stars were taken from own ESO and Calar Alto observations, the Torres \& Massey (1987) atlas, and the IUE archive. The interstellar reddening is derived from fitting the spectral energy distribution. The line spectra of all WC 4-6 stars look amazingly similar. They all fall into the regime of parameter degeneracy $\left(T_{*}>90 \mathrm{kK}\right)$, whereas the WC 7 stars are less hot $\left(T_{*}<80 \mathrm{kK}\right)$. The coarse fits with grid models yield $\log R_{\mathrm{t} 100 \mathrm{kK}}=0.4$ with only small scatter, which implies $\log \left(L / \mathrm{L}_{\odot}\right)-\frac{4}{3} \log \left(\dot{M} /\left(\mathrm{M}_{\odot} \mathrm{yr}^{-1}\right)=11.9\right.$. Only four of the program stars have known distances, allowing for an estimate of their luminosity: $\log \left(L / \mathrm{L}_{\odot}\right)=5.4,5.1,5.2,5.3$ for WR 23 , WR 111, WR 154 and WR 68, respectively. The helium/carbon abundance ratio is determined (cf. Figure 2). Again, most program stars are very similar and give a good fit with He:C $=55: 40$ (by mass) as chosen for the grid. Only four stars show slightly less carbon: WR 5 and WR 14: 30\%; WR 17 and WR68: $\lesssim 30 \%$.

\section{References}

Gräfener, G., Koesterke, L., Hamann, W.-R. 2002, A\&A 387, 244

Hamann, W.-R., Koesterke, L. 1998, A\&A 335, 1003

Torres, A.V., Massey, P. 1987, ApJS 65, 59 